\title{
Artigo Original / Original Paper \\ Riqueza e endemismo de Lauraceae no Paraná: aspectos fitogeográficos e áreas prioritárias para a conservação
}

\author{
Richness and endemism of Lauraceae in Paraná: Phytogeographical aspects \\ and priority areas for conservation
}

Marcelo Leandro Brotto ${ }^{1,4}$, Alexandre Uhlmann ${ }^{3}$, Christopher Thomas Blum ${ }^{1}$ \& Carlos Vellozo Roderjan ${ }^{2}$

\begin{abstract}
Resumo
Lauraceae está entre as três principais famílias do componente arbóreo das florestas no Paraná, sendo elemento frequente nos estudos fitossociológicos e biogeográficos. Apesar disso, a família continua insuficientemente conhecida no estado. Nossos objetivos foram: reconhecer as espécies de Lauraceae no Paraná, relacionando-as com as unidades fitogeográficas; mapear as regiões com maior riqueza de espécies e concentração de endemismos; identificar áreas de complementaridade e compará-las com as áreas prioritárias para conservação. Para isso, nós utilizamos registros de coleções de 23 herbários nacionais, totalizando 3.332 espécimes analisados, plotando suas coordenadas no mapa fitogeográfico do estado. Constatamos que no Paraná ocorrem 76 espécies, das quais onze são citadas pela primeira vez no estado. A Floresta Ombrófila Densa abrange o maior número de espécies ( 59 spp.), seguida pela Floresta Ombrófila Mista (31 spp.), Floresta Estacional Semidecidual (23 spp.), Savana (9 spp.) e Estepe (2 spp.). As regiões da Serra do Mar e Vale do Ribeira apresentam a maior riqueza e endemismo. O Parque Estadual das Lauráceas é uma unidade de conservação estratégica, pois abriga dois terços das espécies paranaenses, com sete endêmicas.
\end{abstract}

Palavras-chave: áreas prioritárias, biogeografia, complementaridade, endemismo, Mata Atlântica.

\begin{abstract}
Lauraceae are among the three main families of the arboreal component of the forests in Paraná, being a frequent element in phytosociological and biogeographic studies. In spite of this, the family remains insufficiently known in the state. Our aims were: to recognize the species of Lauraceae in Paraná, relating them to the phytogeographic units; map the regions with the greatest species richness and concentration of endemisms; identify complementarity areas and compare them with the priority areas for conservation. We used collection records from 23 national herbaria, totaling 3,332 specimens analyzed, plotting their geographic coordinates onto the phytogeographic map of the state. We found that there are 76 species in Paraná, of which eleven are cited for the first time in the state. The Atlantic Rainforest contains the largest number of species (59 spp.), followed by the Araucaria Rainforest (31 spp.), Semideciduous Forest (23 spp.), Savanna (9 spp.), and Grasslands (2 spp.). The Serra do Mar and Ribeira Valley regions have the highest concentration of species, as well as the highest index of endemism. The Lauráceas State Park is a strategic conservation unit, because it houses two thirds of the species of Paraná, including seven endemic. Key words: priority areas, biogeography, complementarity, endemism, Atlantic Forest.
\end{abstract}

\footnotetext{
${ }^{1}$ Museu Botânico Municipal de Curitiba, R. Ostoja Roguski s/n, CP 1142, Curitiba, PR, Brasil.

${ }^{2}$ Universidade Federal do Paraná, Depto. Ciências Florestais, Av. Prefeito Lothário Meissner 632, Jardim Botânico, 80210-170, Curitiba, PR, Brasil.

${ }^{3}$ EMBRAPA Pesca e Aquicultura, prolongamento da Av. NS 10, cruzamento com a Av. LO 18, sentido Norte, loteam. Água Fria, C.P. 90, Palmas, TO, Brasil.

${ }^{4}$ Autor para correspondência: mabrotto@smma.curitiba.pr.gov.br
} 


\section{Introdução}

Lauraceae abrange árvores e arbustos, exceto Cassytha que é hemiparasita, com cerca de 50 gêneros e 2.500-3.500 espécies; é predominantemente tropical, tendo como principais centros de diversidade os trópicos da América e da Ásia (Rohwer 1993; Van der Werff \& Richter 1996). Na região Neotropical, a família se destaca especialmente nas florestas de terras baixas ou em altitudes intermediárias e sobre solos pobres (Gentry 1988). No entanto, sua diversidade absoluta pode ser maior na floresta úmida de terras baixas (Rohwer 1993).

No Brasil, ocorrem 24 gêneros e 441 espécies, com alta riqueza em dois biomas: Amazônia, onde são referidas 242 espécies, das quais 198 são endêmicas; e Mata Atlântica, com 205 espécies, sendo 128 endêmicas (Quinet et al. 2015). Apesar do estado do Paraná possuir apenas 2,5\% do território brasileiro (Maack 2002), abriga 12 gêneros e 69 espécies de Lauraceae e, portanto, cerca de $16 \%$ das espécies que ocorrem no país (Quinet et al. 2015). Diversos autores já enfatizaram a importância de representantes da família no estado, principalmente na composição das formações florestais e em sua exploração para a indústria da madeira (Hoehne 1930; Veloso 1962; Hueck 1972; Inoue et al. 1984; Klein 1984; Maack 2002; Roderjan et al. 2002; Scheer \& Blum 2011). Entretanto, nesses trabalhos são citados apenas os táxons mais comuns, de maior importância na estrutura das florestas, ou então, aqueles com interesse econômico.

Os vários estudos taxonômicos de Lauraceae no Paraná realizados a partir da década de 1950 (Vattimo-Gil 1956a, 1956b, 1962, 1979; Zanon et al. 2009; Brotto et al. 2009, 2013) têm se mostrado insuficientes para o reconhecimento de todas as espécies que existem nesse estado. Um exemplo disso foi a descoberta de dois táxons novos em anos recentes (Brotto et al. 2010; Brotto \& Baitello 2012). Por essa razão, a execução de mais estudos taxonômicos e biogeográficos é necessária para permitir a identificação dos espécimes, a revisão das coleções nos herbários e as atualizações nomenclaturais, além de contribuir com o conhecimento sobre as unidades fitogeográficas e suas espécies.

Em estudo relativamente recente que tratou da distribuição das espécies exclusivamente no território paranaense, baseado na compilação de dados de 58 levantamentos fitossociológicos no Paraná, Scheer \& Blum (2011) relataram que
Lauraceae são uma das três principais famílias do compartimento arbóreo de suas florestas, sendo a segunda mais rica em espécies nas Florestas Ombrófilas Densa (39 spp.) e Mista (23 spp.), atrás apenas de Myrtaceae, e em terceiro lugar na Floresta Estacional Semidecidual (19 spp.), atrás de Fabaceae e Myrtaceae. Tendência similar foi demonstrada por Oliveira-Filho \& Fontes (2000), posicionando Lauraceae entre as famílias com maior número de táxons nas florestas ombrófilas em relação às florestas estacionais do Bioma Mata Atlântica, com destaque nas maiores altitudes.

É crescente a demanda por informações atualizadas sobre táxons e suas áreas de distribuição, impulsionada especialmente pela produção da Lista de Espécies da Flora do Brasil (BFG 2018) e pela Lista de Espécies da Flora Brasileira Ameaçada de Extinção (Martinelli \& Moraes 2013; MMA 2014). Atualmente no Paraná existe uma demanda pelas mesmas informações, particularmente para subsidiar a atualização da lista estadual de espécies ameaçadas de extinção, publicada há mais de 20 anos (Hatschbach \& Ziller 1995). Além disso, trabalhos recentes têm dispendido esforços na tentativa de classificar com mais clareza as tipologias florestais na porção subtropical do Bioma Mata Atlântica, bem como, detectar riqueza de espécies e endemismos, que são informações de vital importância para a conservação (Marques et al. 2011; Rezende et al. 2014, 2015a, 2015b; Oliveira-Filho et al. 2015).

Buscando contribuir com informações atualizadas sobre o tema e considerando que as espécies de Lauraceae estão entre as mais importantes na composição florística e na estrutura das florestas no estado do Paraná, nós objetivamos: a) listar todas as espécies de Lauraceae existentes no Paraná e relacionar suas distribuições às unidades fitogeográficas; b) descrever como a riqueza de espécies de Lauraceae está distribuída no território paranaense; c) reconhecer as áreas com maior concentração de espécies endêmicas ou com distribuição restrita; d) identificar áreas de complementaridade e compará-las com as áreas prioritárias para conservação.

\section{Material e Métodos}

O estado do Paraná está situado na região Sul do Brasil, entre as coordenadas $22^{\circ} 29^{\prime} 30^{\prime \prime}-26^{\circ} 41^{\prime} 00^{\prime \prime}$ $\mathrm{S}$ e $48^{\circ} 02^{\prime} 24^{\prime \prime}-54^{\circ} 37^{\prime} 38^{\prime \prime}$ W. Com área de 199.323 $\mathrm{km}^{2}$, ocupa $2,5 \%$ da superfície total do país (Maack 2002), sendo composto por cinco unidades fitogeográficas. No Bioma Mata Atlântica estão 
contidas a Floresta Ombrófila Densa (Floresta Atlântica), a Floresta Ombrófila Mista (Floresta com Araucária), a Floresta Estacional Semidecidual (Floresta Estacional) e a Estepe (Campos), enquanto que no Bioma Cerrado está contida a Savana (Cerrado). A base cartográfica utilizada no presente estudo é a proposta por Roderjan et al. (2002). No decorrer do texto as três primeiras são referidas como FOD, FOM e FES, respectivamente. O Paraná está sob influência dos tipos climáticos Cfa e Cfb da classificação de Köppen (1948), com a faixa de transição climática entre 600-700 metros de altitude, com o clima $\mathrm{Cfb}$ acima e o Cfa abaixo (Caviglione et al. 2000; Blum et al. 2011; Alvares et al. 2013).

A fonte de dados para nosso estudo se resumiu às coleções de herbários. Os herbários analisados foram os seguintes: ALCB, EFC, ESA, FLOR, FUEL, FURB, HBR, HCF, HFC, HFIE, HRCB, HUCP, HUEM, HUFU, HUPG, ICN, MBM, RB, SP, SPF, SPSF, UNOP, UPCB (acrônimos baseados em Thiers (continuamente atualizado), exceto o sublinhado que não está indexado). Cada registro de herbário (voucher) teve sua identificação confirmada pelo primeiro autor, que inclui também coletas próprias realizadas a partir do ano de 2006 e que foram distribuídas principalmente para os herbários EFC, HUEFS, MBM, MO, NY, RB, SPSF e UPCB. Na Tabela 1 é citado um voucher de referência por espécie.

Os dados contidos nas fichas das exsicatas foram organizados em planilha no programa

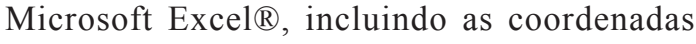
geográficas de cada voucher a fim de mapear a distribuição das espécies. Para isso, foram consideradas as coordenadas originais indicadas nas fichas das exsicatas. Quando não mencionadas, atribuiu-se a coordenada da localidade da coleta e, quando não foi possível identificar a localidade, atribuiu-se a coordenada da sede do município. A acurácia dessas informações nas fichas das exsicatas nos permitiu relacioná-las apenas com as subformações da Floresta Ombrófila Densa (Terras Baixas, Submontana, Montana e Altomontana). Isso não foi possível para as outras unidades fitogeográficas e vegetação pioneira, por falta de detalhamento suficiente para atribuirmos tal relação. O mapeamento foi realizado no programa QGIS 2.10.1-Pisa, no qual foi estabelecida uma grade de quadrículas de $0,5^{\circ} \times 0,5^{\circ}$, que foram utilizadas como unidades amostrais para a quantificação do número de espécies e cálculo do índice de constância relativa $(\mathrm{CR})$ proposto por Mueller-Dombois \& Ellenberg (1974). Classificamos as espécies como de distribuição ampla $(\mathrm{CR}>80 \%)$, intermediária $(20<\mathrm{CR}<80 \%)$ ou restrita $(\mathrm{CR}<20 \%)$, conforme proposto por Scudeller et al. (2001). Para diminuir a distorção no cálculo, excluímos nove quadrículas localizadas em porções limítrofes do território paranaense, por possuírem área reduzida e pela ausência de registro de espécimes, ou seja, das quadrículas sem qualquer registro de coleta, consideramos apenas as B11, C10 e D8. Portanto, utilizamos 84 das 93 quadrículas.

Calculamos também o índice de endemismo (IE) proposto por Usher (1986), conforme demonstrado por Kerr (1997), que nos permitiu avaliar as diferenças de endemismo entre as quadrículas.

Para verificar a similaridade florística entre as unidades fitoecológicas nós realizamos uma análise de agrupamento através do método UPGMA, a partir da matriz de presença e ausência de espécies (Tab. 1), utilizando o coeficiente de similaridade de Sørensen como medida de distância, o que é recomendável para dados qualitativos (Legendre \& Legendre 2012). O cálculo foi realizado no programa Fitopac 2.1.

Para determinar os locais ótimos para a conservação in situ do maior número possível de espécies realizamos uma análise de complementaridade baseada no algoritmo proposto por Rebelo (1994). A partir do resultado, repetimos o procedimento agrupando quadrículas contíguas de mesma formação fitogeográfica e conjunto de espécies. Esta estratégia permitiu a detecção de áreas complementares levando-se em consideração as divisões fitofisionômicas e os limites naturais dos diferentes ambientes no estado, segundo Maack (2002) e Roderjan et al. (2002), levando a uma comparação mais lógica com o mapa de áreas prioritárias para conservação propostas no estado do Paraná (IAP 2014; MMA 2007).

\section{Resultados}

Ao todo nós analisamos 3.332 espécimes depositados em 23 herbários nacionais. A partir desses materiais podemos afirmar que a família Lauraceae está representada no estado do Paraná por 76 espécies, distribuídas em 11 gêneros (Tab. 1). O maior gênero em número de táxons é Ocotea (37 spp.), seguido por Nectandra (14 spp.), Aiouea (9 spp.), Cryptocarya (5 spp.), Persea (4 spp.), Aniba (2 spp.), Beilschmiedia, Cassytha, Endlicheria, Licaria e Rhodostemonodaphne (1 sp.). 


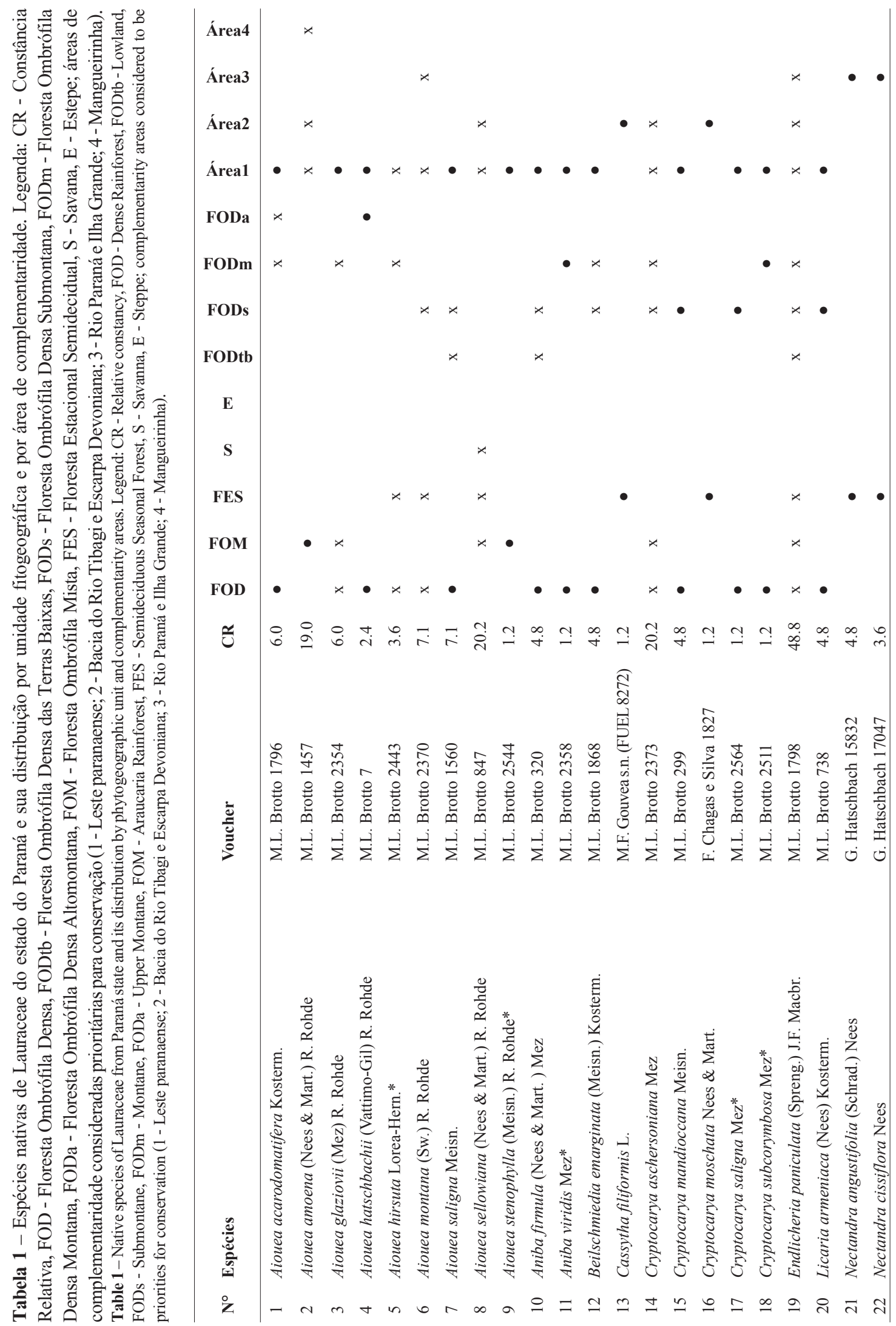




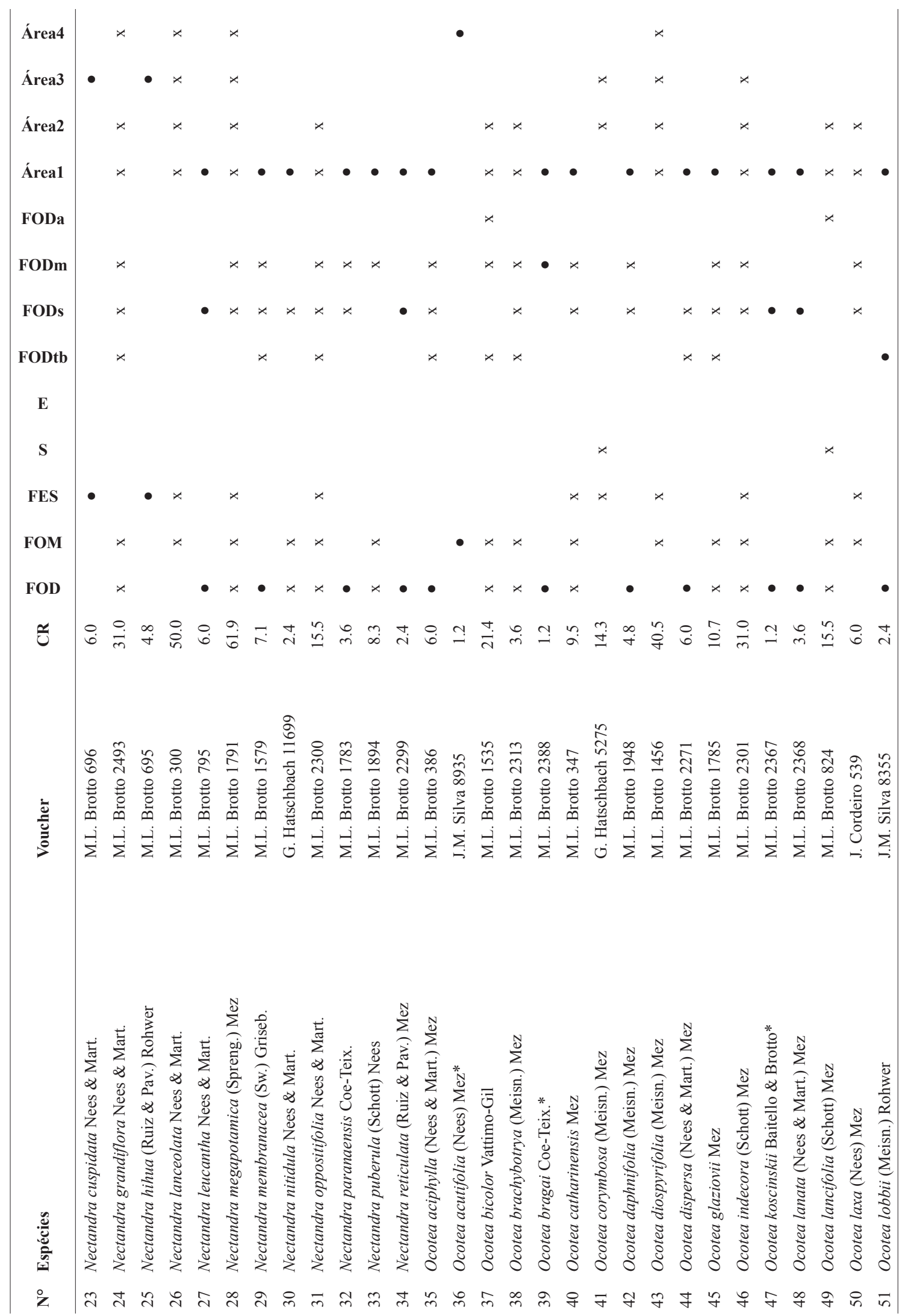




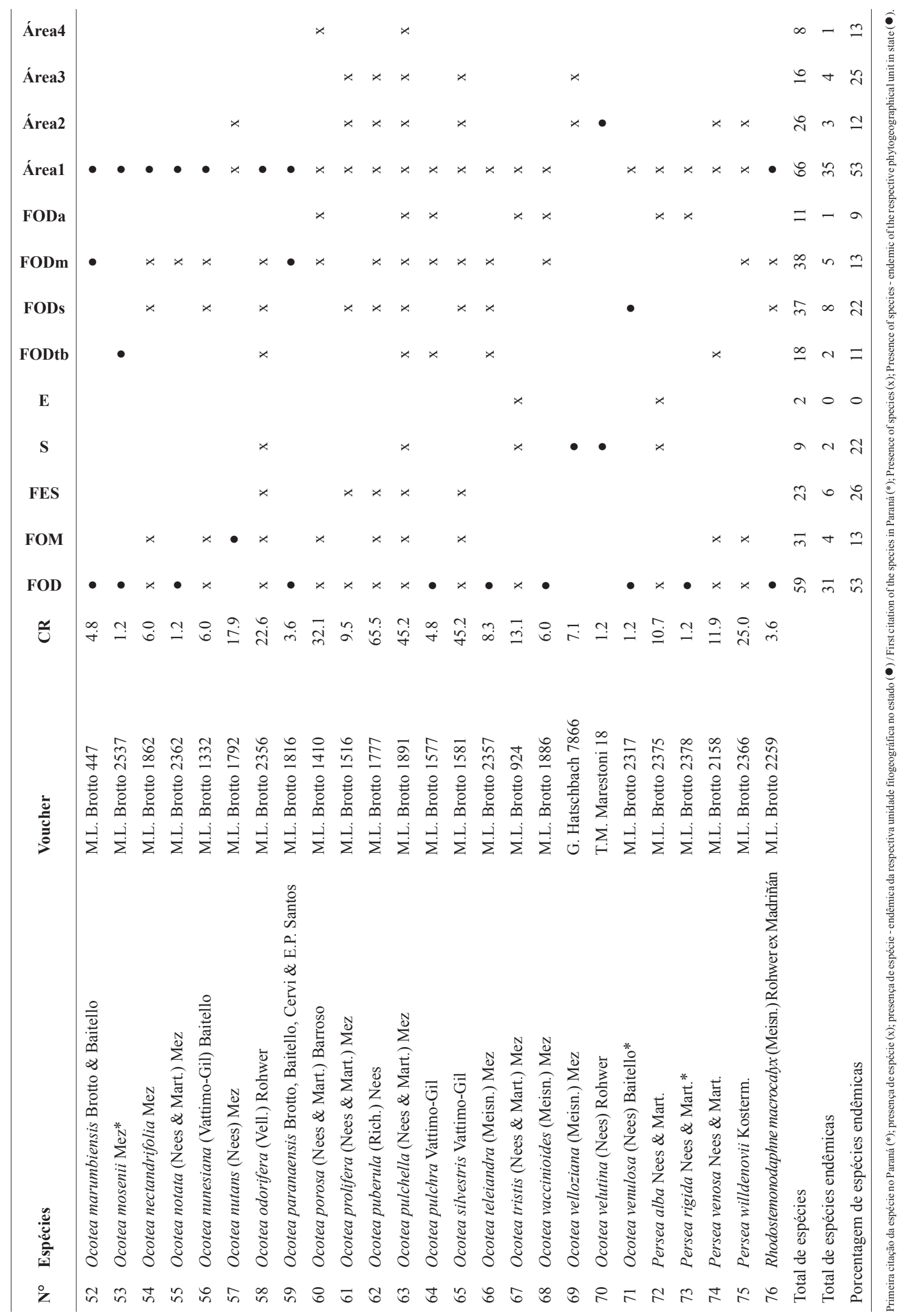


Nós reconhecemos onze espécies que são citadas pela primeira vez no estado: Aiouea hirsuta Lorea-Hern., Aiouea stenophylla (Meisn.) R. Rohde, Aniba viridis Mez, Cryptocarya saligna Mez, Cryptocarya subcorymbosa Mez, Ocotea bragai Coe-Teix. e Persea rigida Nees \& Mart. foram coletadas no Parque Estadual das Lauráceas ou em sua zona de amortecimento (quadrículas F12 e E13, Fig. 1); Ocotea koscinskii Baitello \& Brotto e Ocotea venulosa (Nees) Baitello foram encontradas na RPPN Salto Morato (F14, Fig. 1); Ocotea mosenii Mez foi encontrada na Área de Proteção Ambiental de Guaraqueçaba (F14, Fig. 1); e Ocotea acutifolia (Nees) Mez foi registrada no município de Mangueirinha, sul do Paraná (H6, Fig. 1). Esta última já era citada no estado, mas o reconhecimento se baseava em identificações equivocadas de espécimes, geralmente sendo confundida com Ocotea bicolor Vattimo-Gil.

Em relação às unidades fitogeográficas, a Floresta Ombrófila Densa apresentou a maior riqueza (59 spp.), seguida pela Floresta Ombrófila
Mista (31 spp.), Floresta Estacional Semidecidual (23 spp.), Savana (9 spp.) e Estepe (2 spp.). Das 74 espécies presentes nas fisionomias florestais, apenas nove (12\%) são comuns aos três tipos de floresta (Fig. 2). O maior número de espécies endêmicas ocorre também na FOD (31 spp.), seguida pela FES (6 spp.), FOM (4 spp.), Savana (2 spp.) e nenhuma na Estepe. No âmbito da FOD, a maior riqueza é encontrada na formação Montana (38 spp.), seguida pela Submontana (37 spp.), Terras Baixas (18 spp.) e Altomontana (11 spp.), sendo que o maior número de endêmicas está na formação Submontana (8 spp.) (Tab. 1).

A análise de agrupamento revelou a formação de dois grupos; o grupo 1 composto por vegetação de maior porte, que inclui a FES, FOM e as formações de Terras Baixas, Submontana e Montana da FOD; e o grupo 2, formado por vegetação de menor porte, incluindo a formação Altomontana da FOD, a Savana e a Estepe (Fig. 3). O valor do coeficiente de correlação cofenética para o dendrograma foi de 0,90 .

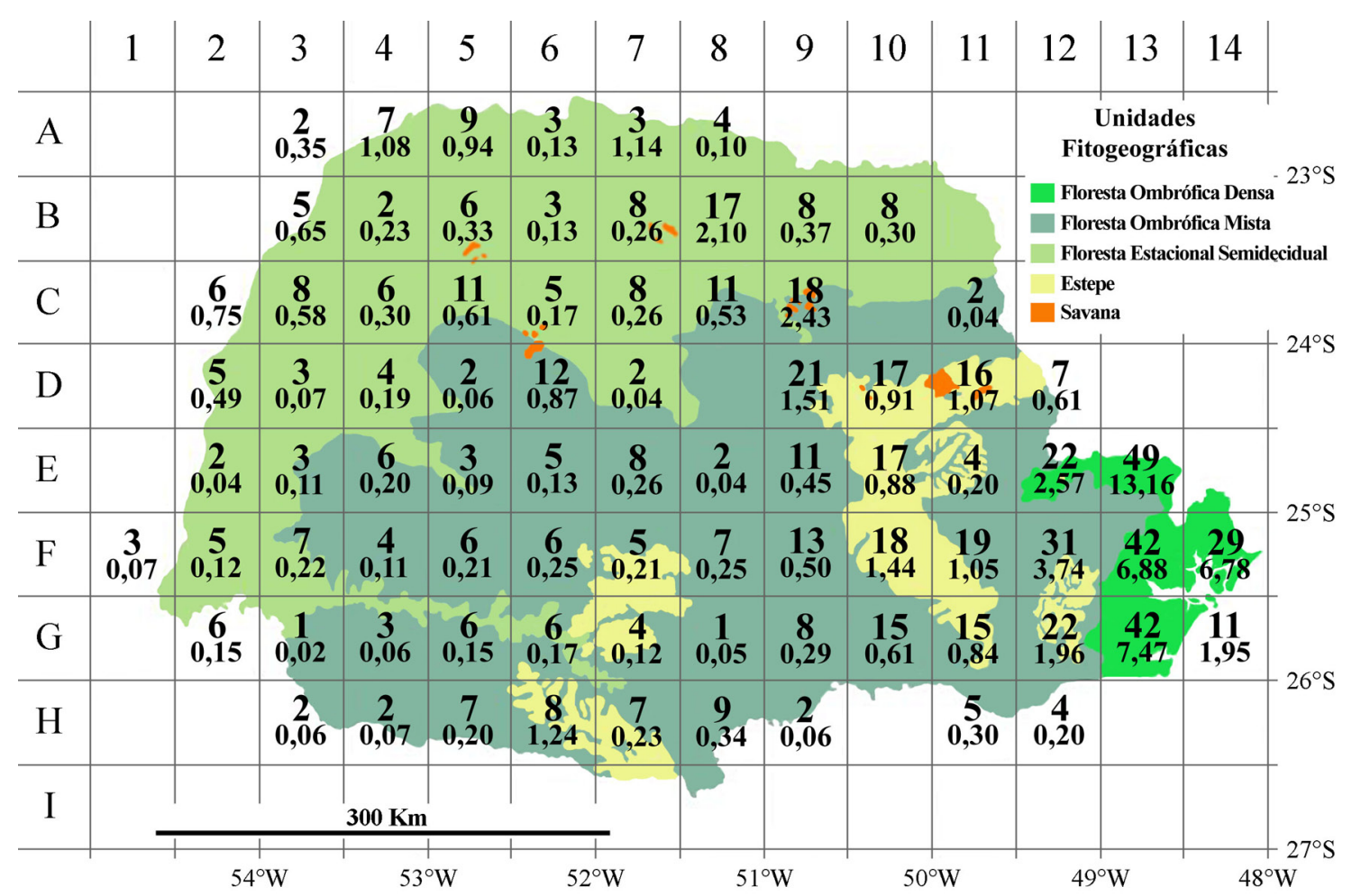

Figura 1 - Riqueza e endemismo de Lauraceae no estado do Paraná por quadrículas de $0,5^{\circ} \times 0,5^{\circ}$. Os números maiores representam a quantidade de espécies e os números menores representam o índice de endemismo (Usher 1986) por quadrícula.

Figure 1 - Richness and endemism of Lauraceae in the state of Paraná by grids $0.5^{\circ} \times 0.5^{\circ}$. The larger numbers represent the number of species and the smaller numbers represent the index of endemism (Usher 1986) by grid. 


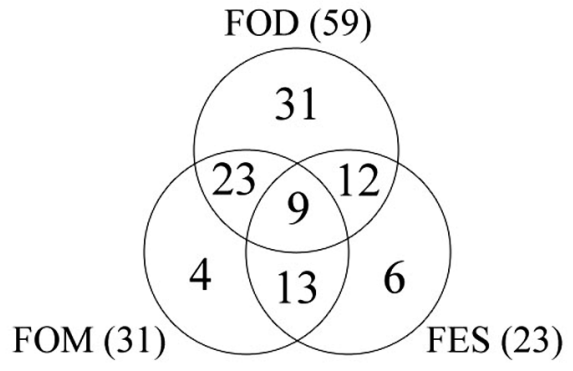

Figura 2 - Diagrama de Venn representando a distribuição das espécies de Lauraceae nas unidades fitogeográficas florestais do Paraná. Legenda: FOD Floresta Ombrófila Densa, FOM - Floresta Ombrófila Mista, FES - Floresta Estacional Semidecidual (FES).

Figure 2 - Venn diagram representing the distribution of species of Lauraceae in the forest domains of Paraná. Legend: FOD - Atlantic Forest, FOM - Araucaria Forest, FES - Semideciduous Forest.

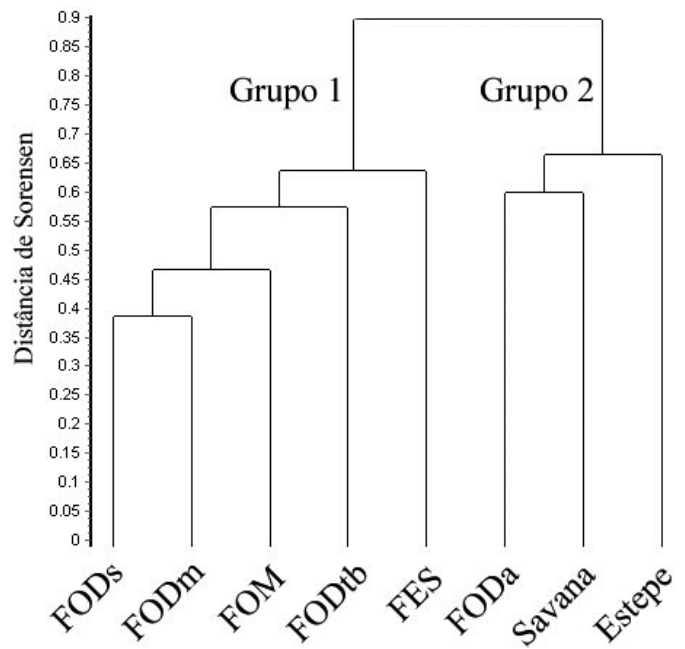

Figura 3 - Dendrograma resultante da análise de agrupamento pelo método UPGMA com utilização do índice de Sørensen como medida de distância, baseado em presença e ausência de espécies de Lauraceae no Paraná. Figure 3 - Dendrogram resulting from cluster analysis by UPGMA method using Sørensen index as distance measure, based on presence and absence of Lauraceae species in Paraná.

Os valores de constância relativa indicam que 61 espécies (80\%) apresentam distribuição restrita $(\mathrm{CR}<20 \%), 15$ espécies $(20 \%)$ têm distribuição intermediária $(20 \% \leq \mathrm{CR}<80 \%)$, e nenhuma foi classificada como de ampla distribuição ( $\mathrm{CR} \geq 80 \%)$ (Tab. 1). Quatorze espécies obtiveram CR igual a 1,2 , o que significa que, foram registradas em apenas uma quadrícula. A região do Vale do Ribeira (F12 e E13) contém sete dessas espécies de ocorrência pontual; a região de Guaraqueçaba (F14) abriga três; o corredor que liga Lupionópolis a São Jerônimo da Serra, no norte do estado (A7, B8 e C9), contém três espécies; e a região de Mangueirinha (H6) tem apenas uma espécie de ocorrência pontual.

A espacialização dos pontos dos espécimes sobre o território resultou em 81 quadrículas com presença de pelo menos um táxon e 12 quadrículas sem qualquer registro (Fig. 1). A maioria destas quadrículas vazias abrange porções limítrofes, onde apenas pequenas extensões das quadrículas se sobrepõem ao território paranaense, o que implica em menor probabilidade de um evento de coleta de espécime. Apenas as quadrículas C10 e D8 estão inteiramente no território e não apresentaram registros.

A maior riqueza de espécies foi registrada em três quadrículas que englobam as regiões da Planície Litorânea, Serra do Mar e Vale do Ribeira (E13, G13, F13) (Fig. 1). Estas três, juntas com F14, também alcançaram os maiores valores do Índice de Endemismo (IE). O destaque é a região do Parque Estadual das Lauráceas (E13), que reúne 49 espécies, sendo que sete são encontradas apenas nessa quadrícula - Aiouea hirsuta, Aniba viridis, Cryptocarya saligna, C. subcorymbosa, Ocotea bragai, O. notata e Persea rigida - o que fez o IE alcançar o valor de 13,16. A quadrícula F14 $(\mathrm{IE}=6,78)$ também teve três espécies exclusivas, O. mosenii que foi registrada na APA de Guaraqueçaba, além de $O$. koscinskii e $O$. venulosa que foram registradas na RPPN Salto Morato.

No Primeiro Planalto do Paraná as quadrículas F12 e G12, junto com a quadrícula E12 do Vale do Açungui/Ribeira, apresentaram a maior riqueza de espécies após as anteriores. No Segundo e no Terceiro Planaltos do Paraná a maior riqueza está concentrada na bacia do Rio Tibagi, desde sua nascente na região de Ponta Grossa (F10), passando pelo terço médio na região de Telêmaco Borba (D9), até seu terço final na região de Londrina (B8). Os destaques são as regiões de Telêmaco Borba (D9) que somou 21 spp. e de São Jerônimo da Serra (C9) que obteve o maior valor de IE $(2,43)$.

A análise de complementaridade resultou em quatro áreas que comtemplam as 76 espécies de Lauraceae no Paraná (Tab. 1). O Leste paranaense (área 1), com superfície aproximada de 15.200 $\mathrm{km}^{2}$, reúne 66 espécies, das quais 35 estão restritas, abrigando quase a totalidade da FOD e uma porção 
da FOM ao redor e ao norte de Curitiba. A Bacia do Rio Tibagi e Escarpa Devoniana (área 2), com superfície aproximada de $23.400 \mathrm{~km}^{2}$, reúnem 26 espécies, sendo apenas três restritas, abrigando parte da FES, FOM, Estepe na bacia do Tibagi, além de alguns relictos de Savana na Escarpa Devoniana. O Rio Paraná e Ilha Grande (área 3), com superfície aproximada de $11.400 \mathrm{~km}^{2}$, reúnem 16 espécies, sendo apenas quatro restritas a ela, abrigando parte da FES no noroeste do estado. A região de Mangueirinha (área 4), com superfície aproximada de $2.800 \mathrm{~km}^{2}$, reúne 8 espécies, sendo apenas uma restrita a ela, abrigando FOM no sul do estado (Fig. 4).

As quatro áreas de complementaridade coincidem em parte com as áreas estratégicas para a conservação da biodiversidade no âmbito estadual (IAP 2014), como mostra a Figura 4. No âmbito federal (MMA 2007), a área 1 compreende uma zona que engloba onze áreas prioritárias (Ma 116, Ma 135, Ma 144, MaZc 108, MaZc 110, MaZc 112, MaZc 133, MaZc 115, MaZc 122, MaZc 123 e MaZc 126); a área 2 engloba ou sobrepõe parcialmente dez áreas prioritárias (Ma 160, Ma 161, Ma 165, Ma 181, Ma 199, Ma 201, Ma 204, Ma 228, Ma 241 e Ma 258); a área 3 sobrepõe parcialmente cinco áreas prioritárias (Ma 139, Ma 166, Ma 176, Ma 254 e Ma 260); a área 4 também sobrepõe parcialmente apenas uma (Ma 106).

\section{Discussão}

Problemas com determinação em nível de espécie ainda estão entre as maiores dificuldades nas análises de vegetação no Bioma Mata Atlântica, principalmente na FOD (Scudeller et al. 2001). O incremento de onze novos registros na Flora do Paraná atesta a importância de buscas dirigidas a grupos de taxonomia complexa como Lauraceae. A quantidade de espécies registrada pelo nosso estudo representa cerca de $17 \%$ do total reconhecido por Quinet et al. (2015) para o Brasil, mas com algumas diferenças na lista de táxons apresentada por estes autores para o estado do Paraná. Excluindo Ocotea velloziana e $O$. velutina que são exclusivas dos relictos de Savana no Paraná (Brotto et al. 2013), as 74 restantes representam 36\% das 205

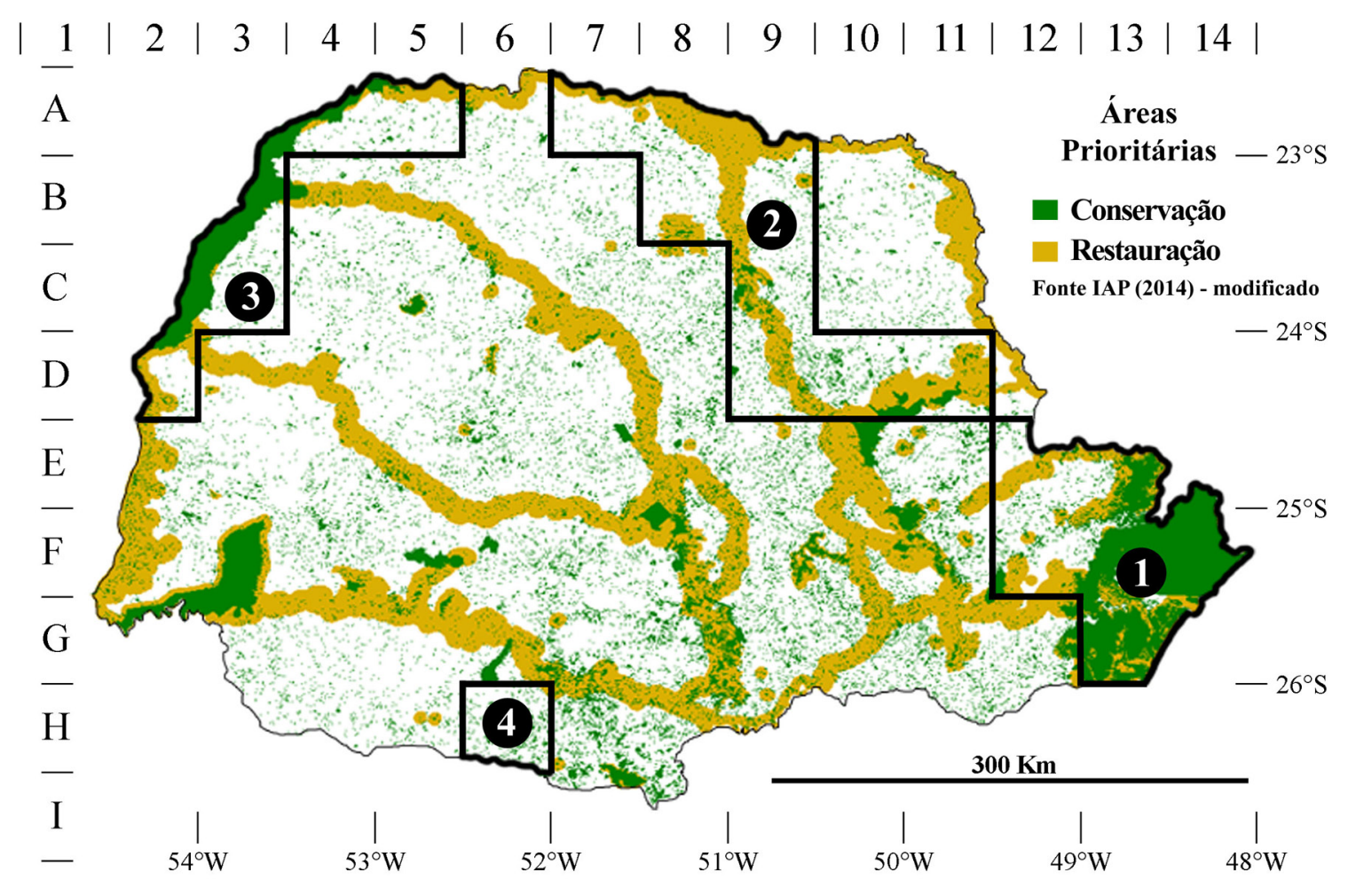

Figura 4 - Mapa das áreas estratégicas para a conservação da biodiversidade no Paraná com a sobreposição das áreas de complementaridade prioritárias para conservação de Lauraceae (Fonte: IAP 2014 - modificado).

Figure 4 - Map of strategic areas for biodiversity conservation in Paraná, with the overlapping areas of priority complementarity areas for conservation of Lauraceae (Font: IAP 2014 - modified). 
espécies do Bioma Mata Atlântica (Quinet et al. 2015). O menor número de espécies em relação ao estado de São Paulo (Baitello et al. 2003) parece seguir a tendência geral das florestas subtropicais brasileiras, onde se observa um declínio na riqueza de espécies arbóreas com o aumento da latitude (Oliveira-Filho et al. 2015). Embora seja uma tendência esperada para essa família de caráter tropical (Rohwer 1993; Van der Werff \& Richter 1996), nós reconhecemos que são muitas as variáveis envolvidas na diferenciação das floras entre os estados brasileiros.

Os maiores valores de riqueza e endemismo foram registrados na porção leste do estado, inserida no contexto da Floresta Ombrófila Densa e ecossistemas associados, e que compreende a Planície Litorânea, Serra do Mar e Vale do Ribeira (E13, F13, G13, F14). Essa região é considerada como área prioritária para a conservação de alguns grupos de animais e plantas (Löwenberg Neto \& Carvalho 2004; Murray-Smith et al. 2008). A alta diversificação ambiental, que permite o desenvolvimento de várias formações vegetacionais (Roderjan et al. 2002), parece ser a explicação mais plausível para a grande concentração de táxons nessa região.

Já o ordenamento das subformações da FOD constituiu um gradiente de maior riqueza da Montana, para a Submontana, Terras Baixas e Altomontana, concordando com os resultados de Scheer \& Blum (2011). Ocotea repetiu o mesmo ordenamento, diferindo parcialmente da ordem apresentada por estes autores, que foi Montana (15 spp.), Submontana (9 spp.), Altomontana (5 spp.) e Terras Baixas (3 spp.). Tendência semelhante foi detectada por Kamimura et al. (2017) em um gradiente de 10-1.093 m de altitude na Serra do Mar de São Paulo, onde houve aumento da riqueza de Lauraceae com aumento da elevação. Vemos, portanto, que essa tendência se inverte na FOD Altomontana paranaense. Supomos que a justificativa para isso possa ser atribuída às condições mais restritivas de solo e clima que ocorrem acima dos $1.200 \mathrm{~m}$, altitude que delimita essa subformação no Paraná (Roderjan et al. 2002). A preferência das Lauraceae por elevações intermediárias em torno dos $1.000 \mathrm{~m}$ de altitude se repete em outras regiões tropicais e subtropicais, como nos Andes, em Madagascar, na Austrália (Gentry 1988), na Indonésia (Culmsee \& Pitopang 2009), e na China (Zhang et al. 2012; Zhu et al. 2015).

No Primeiro Planalto do Paraná, as quadrículas F12 e G12 apresentam a maior riqueza de espécies após as anteriores (E13, F13, G13), com queda de até $55 \%$ no número de espécies e de até $85 \%$ no IE. Essa elevada riqueza na área de abrangência da FOM se deve à contribuição de alguns táxons da FOD. Entretanto, a queda mais acentuada no IE indica que várias espécies estão relacionadas a nichos específicos na FOD, o que é uma tendência reconhecida (Scudeller et al. 2001), e que apenas algumas têm capacidade para ocupar a zona de ecótono com a FOM (Brotto et al. 2013). Isso foi observado para alguns táxons típicos de FOD que têm distribuição ampliada para os contrafortes ocidentais da Serra do Mar, ou mesmo, avançando sobre o Primeiro Planalto, como Aiouea acarodomatifera, Nectandra puberula, Ocotea marumbiensis, $O$. nectandrifolia, O. nunesiana, O. paranaensis e O. vaccinioides (Brotto et al. 2013). Além dessas, Ocotea catharinensis, que é uma das espécies dominantes nas encostas da Serra do Mar (Roderjan et al. 2002), tem três registros em FES no interior do estado, demonstrando padrão idêntico ao relatado no estado de São Paulo (Baitello et al. 2003).

No Segundo e no Terceiro Planaltos do Paraná, a maior riqueza de espécies está concentrada na bacia do Rio Tibagi. Essa região é marcada por alta heterogeneidade ambiental, sendo influenciada por transições climáticas, topográficas e vegetacionais, formando um ecótono entre a FES, FOM, Savana e Estepe (Carmo \& Assis 2012). Com frequência, o número de espécies é maior no ecótono do que nas comunidades que o margeiam (Odum 2004), tendência esta que já foi comprovada pela alta diversidade de espécies arbóreas encontrada no Parque Estadual do Guartelá (Carmo \& Assis 2012), inclusive para a família em estudo. Esse aspecto é particularmente notável nas quadrículas de Telêmaco Borba (D9) e de São Jerônimo da Serra (C9), que apresentaram o maior valor de riqueza e o maior de endemismo, respectivamente.

$\mathrm{O}$ ordenamento das unidades fitoecológicas segundo a sua riqueza demonstrou um claro gradiente leste-oeste, havendo, maior quantidade de espécies nas zonas proximais ao oceano, fato comumente observado em outros estudos (OliveiraFilho \& Fontes 2000; Scheer \& Blum 2011; Higuchi et al. 2013). A alta diversidade da família no leste parece estar relacionada com a barreira geográfica natural da Serra do Mar, que retém as massas de ar quentes e úmidas do oceano Atlântico, ocasionando chuvas relativamente intensas e bem distribuídas ao longo do ano (Roderjan et al. 2002). 
Esse é um padrão genérico no Neotrópico, onde a precipitação total anual e a intensidade da estação seca estão fortemente correlacionadas, a tendência é de que a diversidade aumente linearmente com a precipitação (Gentry 1988). Especificamente nas florestas subtropicais dos Biomas Mata Atlântica e Pampa, a riqueza de espécies arbóreas diminui substancialmente com o aumento da latitude, das florestas ombrófilas em direção às estacionais, e também na cumeada das serras e em praias arenosas (Oliveira-Filho et al. 2015).

Essas tendências são facilmente observadas em nosso estudo. A Figura 1 mostra que a maior média de espécies por quadrícula é verificada na metade oriental do estado, a leste do meridiano $51,5^{\circ} \mathrm{W}$, com o valor de 12 espécies por quadrícula, contra apenas 4,8 por quadrícula na metade ocidental. Isso é resultado da alta concentração de espécies endêmicas da FOD, mas também de um gradiente florístico sutil ao longo das áreas da FES e da FOM. Nós percebemos que a riqueza de espécies na metade ocidental da FOM é menor que na metade oriental e que na FES a quantidade de espécies diminui progressivamente do nordeste para o noroeste, com os menores valores ocorrendo no oeste/sudoeste do Paraná. Portanto, presumimos que as espécies de Lauraceae respondem positivamente à heterogeneidade bioclimática e às rotas migratórias estabelecidas no sul do Brasil (Higuchi et al. 2013).

Maack (2002) foi o primeiro a relatar essa alternância florística ao longo da FES paranaense, descrevendo que ao sul do divisor de águas IvaíPiquiri, a mata pluvial-tropical transforma-se gradativamente em subtropical sem limite climático ou biológico nítido. Nós confirmamos que esse padrão também se aplica às Lauraceae ao constatar que no noroeste do Paraná, ao norte do Rio Piquiri, se distribuem as espécies essencialmente tropicais como Nectandra cissiflora, N. cuspidata, N. hihua, Ocotea velloziana (Moraes 2005), Ocotea corymbosa (Brotto et al. 2013), e $O$. prolifera (Assis 2009). Ao sul do Rio Piquiri, em zona subtropical, se estendem apenas as espécies de maior plasticidade ambiental como Endlicheria paniculata, Nectandra lanceolata, $N$. megapotamica, Ocotea diospyrifolia, O. puberula, O. pulchella e O. silvestris (Baitello et al. 2003).

Mesmo sendo uma família tipicamente tropical (Rohwer 1993; Van der Werff \& Richter 1996), a maior similaridade florística se deu entre a FOD e a FOM do que entre a FOD e a FES. O diagrama de Venn mostrou que a FOM compartilha
$74 \%$ de suas Lauraceae com a FOD e $42 \%$ com a FES. Isso difere da tendência geral para todas as famílias no sul do Brasil, onde a maior diferença na composição das espécies é observada entre as florestas costeiras a leste da Serra do Mar e Serra Geral (florestas ombrófilas e nebulares) e as florestas do interior a oeste dessa barreira geográfica (florestas mista e estacional) (Oliveira-Filho et al. 2015). Para Higuchi et al. (2013), que também analisaram todas as famílias no sul do Brasil, a similaridade florística entre a FES e a FOM, decorrente da maior permeabilidade de espécies entre essas unidades fitoecológicas, é atribuída à ausência de uma grande barreira geográfica e climática que as isole. Entretanto, é justamente a Serra do Mar que reúne, em zona climática Cfb (Köppen 1948), as Lauraceae em comum das florestas ombrófilas. Todas as 23 espécies que a FOM compartilha com a FOD ocorrem na subformação Montana e/ou Altomontana. Nessa região, a isoterma da temperatura média de 13 ${ }^{\circ} \mathrm{C}$ no mês mais frio, estimada para a cota dos 800 m s.n.m., coincide com o limite inferior da Montana (Blum et al. 2011). Ou seja, as espécies em comum experimentam, acima dessa altitude, a mesma condição climática de baixas temperaturas e ocorrência de geadas, tanto na porção superior das montanhas da Serra do Mar, quanto nos planaltos do interior.

A separação entre a floresta estacional e as florestas ombrófilas foi reforçada pela análise de agrupamento, inclusive, apresentando maior similaridade entre a FOD das Terras Baixas com a FOM, do que entre a FOM com a FES. O mesmo padrão foi verificado no estado de Santa Catarina, considerando espécies arbóreas e arbustivas de todas as famílias, por meio da aplicação do método WPGMA e distância de Sørensen, cujo resultado mostrou haver maior similaridade entre as formações da FOM e da FOD do que entre qualquer uma delas com a FES (Rezende et al. 2015a). Mas, acima de tudo, a grande separação entre os dois grupos está ligada ao porte da vegetação, com florestas reunidas no grupo 1 e nanoflorestas com campos no grupo 2 . Essa surpreendente similaridade entre as matinhas nebulares, os campos e o cerrado, deve ser tomada com cautela, pois, há de se considerar que Ocotea lancifolia, O. pulchella, O. tristis e Persea alba fazem parte de complexos de espécies ainda pouco estudados. Rohwer (1986), em sua sinopse de Ocotea sensu lato, já havia definido grupos informais para cada uma dessas três espécies do 
gênero Ocotea, subordinando vários nomes a cada grupo. Kopp (1966), determinou que Persea alba se distribui apenas no sul do Brasil, porém, indicando materiais adicionais para o Rio de Janeiro, Minas Gerais e Goiás. Isso leva a supor que talvez sejam táxons de diferentes linhagens, morfologicamente muito semelhantes, contribuindo para uma falsa similaridade entre as fitofisionomias. Mas, a menos que estudos futuros redefinam a circunscrição desses táxons, a similaridade entre a Estepe, a Savana e a FOD Altomontana, deve ser tomada como verdadeira.

$\mathrm{O}$ número de espécies comuns às três formações florestais é baixo, assim como para as demais famílias no sul do Brasil (Scheer \& Blum 2011; Higuchi et al. 2013; Rezende et al. 2014). Os nove táxons com essa característica apresentaram constância relativa intermediária $(20<\mathrm{CR}<80 \%)$, representando apenas $12 \%$ das espécies florestais. Rezende et al. (2015b), estudando árvores e arbustos de todas as famílias em Santa Catarina, encontraram mais que o dobro $(27,9 \%)$. Isso demonstra que as Lauraceae são particularmente sensíveis às variações ambientais ao longo dos ecossistemas florestais do Bioma Mata Atlântica. Nesta região, as Lauraceae foram melhores ao indicar padrões de diversidade do que as famílias Fabaceae, Myrtaceae e Rubiaceae, demonstrando seu potencial para estudos biogeográficos (Murray-Smith et al. 2008).

Por sua vez, a quantidade de espécies de Lauraceae endêmicas de uma única unidade fitogeográfica é alto (58\%). O número de espécies exclusivas é maior na FOD, seguindo a mesma tendência para as demais famílias no sul do Brasil (Scheer \& Blum 2011; Higuchi et al. 2013). Retirando Ocotea lobbii, que é exclusiva de solos arenosos das Terras Baixas e Restinga, e Aiouea hatschbachii, que ocorre apenas em ambientes altomontanos, todas as outras 41 espécies endêmicas das suas respectivas unidades fitogeográficas ocorrem em hábitats que não são muito severos (i.e., floresta nebular e restinga), concordando com tendência geral das espécies endêmicas subtropicais dos Biomas Mata Atlântica e Pampa (Oliveira-Filho et al. 2015).

No contexto das Lauraceae, uma metodologia relativamente simples aliada a uma fonte de dados básica (coleções de herbários) foram capazes de apontar quatro áreas complementares, e que coincidiram com áreas prioritárias para conservação, tanto no âmbito estadual quanto no federal (MMA 2007; IAP 2014). Em especial destaca-se a região leste que reúne a maior riqueza de espécies e endemismos, também considerada prioritária para a conservação por outros pesquisadores (Löwenberg Neto \& Carvalho 2004; Murray-Smith et al. 2008; Marques et al. 2011).

Cabe a ressalva de que os valores de riqueza e endemismo foram diretamente influenciados por esforços de coleta diferenciados para cada região, como é evidente na figura 1 , onde uma quadrícula com 21 espécies (D9) é vista ao lado de uma sem um registro sequer (D8). Mesmo assim, há de se considerar que análises espaciais amplas, baseadas em dados relativamente grosseiros, permitem investigar regiões potencialmente importantes nas quais podem ser realizados estudos mais detalhados, como para avaliar fragmentação de hábitat, padrões de viabilidade populacional, ou manutenção da diversidade genética (DinizFilho et al. 2009). Estudos como o que avaliou a diversidade genética em populações de três espécies de Ocotea ameaçadas de extinção, apontando quatro populações prioritárias para conservação in situ no Paraná (Martins et al. 2014).

\section{Agradecimentos}

Agradecemos a Michel Mello e Guilherme Vasconcellos do Instituto Ambiental do Paraná a autorização e apoio à pesquisa no P.E. das Lauráceas. Também a João Batista Baitello e Pedro Luís Rodrigues de Moraes a ajuda com o reconhecimento das espécies, e a Fernando Matos a revisão do texto.

\section{Referências}

Alvares AC, Stape JL, Sentelhas PC, Gonçalves JLM \& Sparovek G (2013) Köppen's climate classification map for Brazil. Meteorologische Zeitschrift 22: 711-728.

Assis LCS (2009) Sistemática e filosofia: filogenia do complexo Ocotea e revisão do grupo Ocotea indecora (Lauraceae). Tese de Doutorado. Universidade de São Paulo, São Paulo. 238p.

Baitello JB, Lorea Hernández FG, Moraes PLR de; Esteves R \& Marcovino JR (2003) Lauraceae. In: Wanderley MGL, Shepherd G, Giulietti AM \& Melhem TM (eds.) Flora fanerogâmica do estado de São Paulo. FAPESP/RiMa, São Paulo. Vol. 3, pp. 149-223.

BFG - The Brazil Flora Group (2018) Brazilian Flora 2020: innovation and collaboration to meet Target 1 of the Global Strategy for Plant Conservation (GSPC). Rodriguésia 69: 1513-1527.

Blum CT, Roderjan CV \& Galvão F (2011) O clima e sua influência na distribuição da Floresta Ombrófila Densa na Serra da Prata, Morretes, Paraná. Floresta 41: 589-598. 
Brotto ML, Santos EP \& Baitello JB (2009) Lauraceae no Morro dos Perdidos (Floresta Atlântica), Paraná, Brasil. Rodriguésia 60: 445-459.

Brotto ML, Baitello JB, Cervi AC \& Santos EP (2010) Uma nova espécie de Ocotea (Lauraceae) para o Brasil. Rodriguésia 61: S57-S60.

Brotto ML \& Baitello JB (2012) Uma espécie nova de Lauraceae da floresta atlântica do Brasil. Rodriguésia 63: 579-585.

Brotto ML, Cervi AC \& Santos EP (2013) O gênero Ocotea (Lauraceae) no estado do Paraná, Brasil. Rodriguésia 64: 495-525.

Carmo MRB do \& Assis MA (2012) Caracterização florística e estrutural das florestas naturalmente fragmentadas no Parque Estadual do Guartelá, município de Tibagi, estado do Paraná. Acta Botânica Brasílica 26: 133-145.

Caviglione JH, Kiihl LRB, Caramori PH \& Oliveira D (2000) Cartas climáticas do Paraná. IAPAR, Londrina. Disponível em <http://www.iapar.br/ modules/conteudo/conteudo.php?conteudo $=677>$. Acesso em 9 junho 2017.

Culmsee H \& Pitopang R (2009) Tree diversity in submontane and lower montane primary rain forests in Central Sulawesi. Blumea 54: 119-123.

Diniz-Filho JAF, Bini LM, Oliveira G, Barreto BS, Silva MMFP, Terribile LC, Rangel TFLVB, Pinto MP, Souza NPR, Vieira LCG, Melo AS, Marco Júnior P, Vieira CM, Blamires D, Bastos RP, Carvalho P, Ferreira LG, Telles MPC, Rodrigues FM, Silva DM, Silva Júnior NJ \& Soares TN (2009) Macroecologia, biogeografia e áreas prioritárias para a conservação no cerrado. Oecologia Brasiliensis 13: 470-497.

Gentry A (1988) Changes in plant community diversity and floristic composition on environmental and geographical gradients. Annals of the Missouri Botanical Garden 75: 1-34.

Hatschbach GG \& Ziller SR (1995) Lista vermelha de plantas ameaçadas de extinção no estado do Paraná. SEMA/GTZ, Curitiba. 139p.

Higuchi P, Silva AC, Budke JC, Mantovani A, Bortoluzzi RLC \& Ziger AA (2013) Influência do clima e de rotas migratórias de espécies arbóreas sobre o padrão fitogeográfico de florestas na Região Sul do Brasil. Ciência Florestal 23: 539-553.

Hoehne FC (1930) Araucarilândia: Observações Geraes e Contribuições ao Estudo da Flora e Phytophysionomia do Brasil. Companhia Melhoramentos de São Paulo, São Paulo. 133p.

Hueck K (1972) As florestas da América do Sul: ecologia, composição e importância econômica. Polígono, São Paulo. 476p.

IAP - Instituto Ambiental do Paraná (2014) Mapa: áreas estratégicas para conservação da biodiversidade. GEO, Curitiba. Disponível em <http://www.iap.pr.gov.br/ modules/conteudo/conteudo.php? conteudo $=756>$. Acesso em 26 junho 2019.
Inoue MT, Roderjan CV \& Kuniyoshi YS (1984) Projeto Madeira do Paraná. Fundação de Pesquisas Florestais, Curitiba. 260p.

Kamimura VA, Moares PLR, Ribeiro HL, Joly CA \& Assis MA (2017) Tree diversity and elevational gradient: The case of Lauraceae in the Atlantic Rainforest. Flora 234: 84-91.

Kerr JT (1997) Species richness, endemism, and the choice of areas for conservation. Conservation Biology 11: 1094-1100.

Klein RM (1984) Aspectos dinâmicos da vegetação do sul do Brasil. Sellowia 36: 5-54.

Kopp LE (1966) A taxonomic revision of the genus Persea in the Western hemisphere (Persea-Lauraceae). Memoirs of The New York Botanical Garden 14: $1-117$.

Köppen W (1948) Climatologia: con un studio de los climas de la tierra. Fondo de Cultura Economica, Ciudad de México. 478p.

Legendre P \& Legendre L (2012) Numerical ecology. Elsevier, Amsterdam. 853p.

Löwenberg Neto P \& Carvalho CJB (2004) Análise Parcimoniosa de Endemicidade (PAE) na delimitação de áreas de endemismos: inferências para a conservação da biodiversidade no Sul do Brasil. Natureza e Conservação 2: 58-65.

Maack R (2002) Geografia física do estado do Paraná. $3^{a}$ ed. Imprensa Oficial Paraná, Curitiba. 440p.

Marques MCM, Swaine MD \& Liebsch D (2011) Diversity distribution and floristic differentiaton of the coastal lowland vegetation: implicatons for the conservation of the Brazilian Atlantic Forest. Biodiversity and Conservation 20: 153-168.

Martins EM, Lamont RW, Martinelli G, Lira-Medeiros CF, Quinet A \& Shapcott A (2014) Genetic diversity and population genetic structure in three threatened Ocotea species (Lauraceae) from Brazil's Atlantic Rainforest and implications for their conservation. Conservation Genetics 16: 1-14.

MMA - Ministério do Meio Ambiente (2007) Áreas prioritárias para a conservação, uso sustentável e repartição dos benefícios da biodiversidade brasileira: atualização - portaria MMA Nº 09, de 23 de janeiro de 2007. Disponível em <http://www.mma.gov.br/ estruturas/chm/_arquivos/biodiversidade31.pdf $>$. Acesso em 9 junho 2017.

MMA - Ministério do Meio Ambiente (2014) Portaria

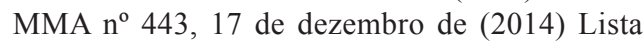
Nacional Oficial de Espécies da Flora Ameaçadas de Extinção. Diário Oficial da União. Imprensa Nacional, Brasília. Seção 1, n 245: 110-121.

Martinelli G \& Moraes MA(2013) Livro vermelho da flora do Brasil. Instituto de Pesquisas Jardim Botânico do Rio de Janeiro / Andrea Jakobsson, Rio de Janeiro. $1100 p$.

Moraes PLR (2005) Sinopse das lauráceas nos estado de Goiás e Tocantins, Brasil. Biota Neotropica 5: 1-18. 
Mueller-Dombois D \& Ellenberg H (1974) Aims and methods of vegetation ecology. John Wiley \& Sons, New York. 547p.

Murray-Smith C, Brummitt NA, Oliveira-Filho AT, Bachman S, Moat J, Nic Lughadha EM \& Lucas EJ (2008) Plant Diversity Hotspots in the Atlantic Coastal Forests of Brazil. Conservation Biology 23: 151-163.

Odum EP (2004) Fundamentos de Ecologia. $7^{\text {a }}$ ed. Fundação Calouste Gulbenkian, Lisboa. 927p.

Oliveira-Filho AT \& Fontes MAL (2000) Patterns of floristic differentiation among Atlantic Forests in Southeastern Brazil an the influence of climate. Biotropica 32: 793-810.

Oliveira-Filho AT, Budke JC, Jarenkow JA, Eisenlohr PV \& Neves DRM (2015) Delving into the variations in tree species composition and richness across South American subtropical Atlantic and Pampean forests. Journal of Plant Ecology 8: 242-260.

Quinet A, Baitello JB, Moraes PLR, Assis L \& Alves FM (2015) Lauraceae. In: Lista de espécies da flora do Brasil. Jardim Botânico do Rio de Janeiro. Disponível em <http://floradobrasil.jbrj.gov.br/jabot/ floradobrasil/FB143>. Acesso em 27 fevereiro 2017.

Rebelo TG (1994) Iterative selection procedures - centres of endemism and optimal placement of reserves. Strelitzia 1: 231-257.

Rezende VL, Eisenlohr PV, Gasper AL, Vibrans AC \& Oliveira-Filho AT (2014) Toward a better understanding of the subtropical Atlantic Forest species richness and frequency of rare species. Acta Botânica Brasílica 28: 382-391.

Rezende VL, Eisenlohr PV, Vibrans AC \& Oliveira-Filho AT (2015a) Humidity, low temperature extremes, and space influence floristic variation across an insightful gradient in the Subtropical Atlantic Forest. Plant Ecology 216: 759-774.

Rezende VL, Oliveira-Filho AT, Eisenlohr PV, Kamino LHY \& Vibrans AC (2015b) Restricted geographic distribution of tree species calls for urgent conservation efforts in the Subtropical Atlantic Forest. Biodiversity and Conservation 24: 1057-1071.

Roderjan CV, Galvão F, Kuniyoshi YS \& Hatschbach GG (2002) As unidades fitogeográficas do estado do Paraná, Brasil. Ciência \& Ambiente 13: 75-92.

Rohwer JG (1986) Prodromus einer Monographie der Gattung Ocotea Aubl. (Lauraceae) sensu lato. Mitteilungen aus dem Institut für Allgemeine Botanik, Hamburg 20: 1-278.

Rohwer JG (1993) Lauraceae. In: Kubitzki K, Rohwer JG \& Bittrich V (eds.) The families and genera of vascular plants. Vol. 2. Springer-Verlag, Berlin. Pp. 366-391.
Scheer MB \& Blum CT (2011) Arboreal diversity of the Atlantic Forest of Southern Brazil: from the beach ridges to the Paraná river. In: Grillo O \& Venora G (eds.) The dynamical processes of biodiversity - case studies of evolution and spatial distribution. InTech. Pp. 109-134.

Scudeller VV, Martins FR \& Shepherd GJ (2001) Distribution and abundance of arboreal species in the atlantic ombrophilous dense forest in Southeastern Brazil. Plant Ecology 152: 185-199.

Thiers B [continuamente atualizado] Index Herbariorum: a global directory of public herbaria and associated staff. Botanical Garden's Virtual Herbarium, New York. Disponível em <http://sweetgum.nybg.org/ih/>. Acesso em 6 fevereiro 2015.

Usher MB (1986) Wildlife conservation evaluation. Chapman \& Hall, London. 394p.

Van der Werff H \& Ritcher HG (1996) Toward and improved classification of Lauraceae. Annals of the Missouri Botanical Garden 83: 409-418.

Vattimo-Gil I (1956a) Nota prévia sobre as espécies de Ocotea Aubl. que ocorrem no estado do Paraná. Arquivos do Serviço Florestal 10: 109-123.

Vattimo-Gil I (1956b) O gênero Ocotea Aubl. no Sul do Brasil. I. Espécies de Santa Catarina e Paraná. Rodriguésia 18-19: 265-350.

Vattimo-Gil I (1962) O gênero Ocotea Aubl. nos estados de São Paulo e Rio Grande do Sul. Apêndice: notas sobre o gênero Cinnamomum Boehm, no Brasil. Arquivos do Jardim Botânico do Rio de Janeiro 17: 199-226.

Vattimo-Gil I (1979) Contribuição ao conhecimento da distribuição geográfica das Lauráceas V. Novas localidades nos estados do Paraná e Rio Grande do Sul. Rodriguésia 31: 135-152.

Veloso HP (1962) Os grandes clímaces do Brasil. I Considerações sôbre os tipos vegetativos da região sul. Memórias do Instituto Oswaldo Cruz 60: 175-213.

Zanon MMF, Goldenberg R \& Moraes PLR (2009) O gênero Nectandra Rol. ex Rottb. (Lauraceae) no estado do Paraná, Brasil. Acta Botânica Brasílica 23: 22-35.

Zhang ZH, Hu G, Zhu JD \& Ni J (2012) Stand structure, woody species richness and composition of subtropical karst forests in Maolan, south-west China. Journal of Tropical Forest Science 24: 498-506.

Zhu H, Yong C, Zhou S, Wang H \& Yan L (2015) Vegetation, floristic composition and species diversity in a tropical mountain nature reserve in Southern Yunnan, SW China, with implications for conservation. Tropical Conservation Science 8: 528-546. 